\title{
Rotation Effects on Unsteady Flow past an Accelerated Isothermal Vertical Plate with Variable Mass Transfer in the Presence of Chemical Reaction of First Order
}

\author{
R. Muthucumaraswamy ${ }^{1 \dagger}$, N. Dhanasekar ${ }^{2}$ and G. E. $\operatorname{Prasad}^{3}$ \\ ${ }^{1}$ Department of Applied Mathematics, Sri Venkateswara College of Engineering, Sriperumbudur 602105, India. \\ ${ }^{2}$ Department of Mathematics, Mohamed Sathak A .J .College of Engineering, Chennai 603 103, India \\ ${ }^{3}$ Department of Mathematics, S.T.Hindu College, Nagercoil, India
}

†Corresponding Author Email: msamy@svce.ac.in

(Received November 1, 2011; accepted March 7, 2013)

\begin{abstract}
An exact analysis of rotation effects on unsteady flow of an incompressible and electrically conducting fluid past a uniformly accelerated infinite isothermal vertical plate with variable mass diffusion, under the action of transversely applied magnetic field has been presented. The plate temperature is raised to Tw and the concentration level near the plate is raised linearly with time. The dimensionless governing equations are solved using Laplace-transform technique. The velocity profiles, temperature and concentration are studied for different physical parameters like magnetic field parameter, chemical reaction parameter, thermal Grashof number, mass Grashof number, Schmidt number, Prandtl number and time. It is observed that the velocity increases with increasing values of thermal Grashof number or mass Grashof number. It is also observed that the velocity increases with decreasing magnetic field parameter or rotation parameter $\Omega$.
\end{abstract}

Keywords: Rotation accelerated, Isothermal, Vertical plate, Heat transfer, Mass diffusion, Magnetic field, Chemical reaction.

\section{NOMENCLATURE}

$\begin{array}{ll}\text { A } & \text { constant } \\ \mathrm{C}^{\prime} & \text { species concentration in the fluid } \\ \mathrm{C} & \text { dimensionless concentration } \\ \mathrm{C}_{\mathrm{w}} & \text { wall concentration } \\ \mathrm{C} \square & \text { concentration far away from the plate } \\ \mathrm{Cp} & \text { specific heat at constant pressure } \\ \mathrm{D} \quad \text { mass diffusion coefficient } \\ \mathrm{Gc} \text { mass Grashof number } \\ \mathrm{Gr} \quad \text { thermal Grashof number } \\ \mathrm{g} \quad \text { accelerated due to gravity } \\ \mathrm{k} \quad \text { thermal conductivity } \\ \mathrm{K} \quad \text { chemical reaction parameter } \\ \mathrm{M} \quad \text { magnetic field parameter } \\ \mathrm{Pr} \quad \text { Prandtl number } \\ \mathrm{Sc} \quad \text { Schmidt number } \\ \mathrm{T} \quad \text { temperature of the fluid near the plate } \\ \mathrm{T}_{\mathrm{w}} \quad \text { temperature of the plate } \\ \mathrm{T} \square \text { temperature of the fluid far away } \\ \\ \mathrm{t}^{\prime} \quad \text { from the plate }\end{array}$

\begin{tabular}{|c|c|}
\hline$t$ & dimensionless time \\
\hline $\mathrm{u}$ & velocity of the fluid in the $\mathrm{x}$-direction \\
\hline $\mathrm{u}_{0}$ & velocity of the plate \\
\hline q & dimensionless velocity \\
\hline X & spatial coordinate along the plate \\
\hline $\mathrm{y}^{\prime}$ & coordinate axis normal to the plate \\
\hline $\mathrm{Z}$ & $\begin{array}{l}\text { dimensionless coordinate axis normal } \\
\text { to the plate }\end{array}$ \\
\hline$\beta$ & $\begin{array}{l}\text { volumetric coefficient of thermal } \\
\text { expansion }\end{array}$ \\
\hline$\beta^{*}$ & $\begin{array}{l}\text { volumetric coefficient of expansion with } \\
\text { concentration }\end{array}$ \\
\hline$\Omega$ & rotation parameter \\
\hline$\mu$ & coefficient of viscosity \\
\hline$v$ & kinematic viscosity \\
\hline$\rho$ & density of the fluid \\
\hline$\tau$ & dimensionless skin-friction $\mathrm{kg}$. \\
\hline$\theta$ & dimensionless temperature \\
\hline$\eta$ & similarity parameter \\
\hline eer & mplementary error fun \\
\hline
\end{tabular}




\section{INTRODUCTION}

The influence of magnetic field on viscous incompressible flow of electrically conducting fluid has its importance in many applications such as extrusion of plastics in the manufacture of rayon and nylon, purification of crude oil, pulp, paper industry, textile industry and in different geophysical cases etc. In many process industries, the cooling of threads or sheets of some polymer materials is of importance in the production line. The rate of cooling can be controlled effectively to achieve final products of desired characteristics by drawing threads, etc. in the presence of an electrically conducting fluid subject to a magnetic field.

MHD plays an important role in agriculture, petroleum industries, geophysics and in astrophysics. MHD flow has application in metrology, solar physics and in motion of earth's core. Also it has applications in the field of stellar and planetary magnetospheres, aeronautics, chemical engineering and electronics. In the field of power generation, MHD is receiving considerable attention due to the possibilities it offers for much higher thermal efficiencies in power plants.

Gupta et al. (1979) studied free convection on flow past a linearly accelerated vertical plate in the presence of viscous dissipative heat using perturbation method. Kafousias and Raptis (1981) extended the above problem to include mass transfer effects subjected to variable suction or injection. Free convection effects on flow past an accelerated vertical plate with variable suction and uniform heat flux in the presence of magnetic field was studied by Raptis et al. (1981). Prasad et al. (2011) have studied finite difference solution of radiative flow past an impulsively started vertical plate with variable heat and mass flux.

MHD effects on flow past an infinite vertical plate for both the classes of impulse as well as accelerated motion of the plate was studied by Raptis and Singh (1981). Mass transfer effects on flow past a uniformly accelerated vertical plate was studied by Soundalgekar (1982). Basant Kumar Jha and Ravindra Prasad (1990) analyzed mass transfer effects on the flow past an accelerated infinite vertical plate with heat sources. Singh (1984) studied MHD flow past an impulsively started vertical plate in a rotating fluid. Rotation effects on hydromagnetic free convective flow past an accelerated isothermal vertical plate was studied by Raptis and Singh (1981).

Chemical reactions can be codified as either heterogeneous or homogeneous processes. This depends on whether they occur at an interface or as a single phase volume reaction. In well-mixed systems, the reaction is heterogeneous, if it takes place at an interface and homogeneous, if it takes place in solution. In most cases of chemical reactions, the reaction rate depends on the concentration of the species itself. A reaction is said to be of first order, if the rate of reaction is directly proportional to the concentration itself. Chambre and Young (1958) have analyzed a first order chemical reaction in the neighborhood of a horizontal plate. Das et al. (1994) have studied the effect of homogeneous first order chemical reaction on the flow past an impulsively started infinite vertical plate with uniform heat flux and mass transfer. Again, mass transfer effects on moving isothermal vertical plate in the presence of chemical reaction studied by Das et al. (1999). The dimensionless governing equations were solved by the usual Laplace-transform technique.

Hence, it is proposed to study the effects of rotation on the hydromagnetic free-convection flow of an incompressible viscous and electrically conducting fluid past a uniformly accelerated infinite isothermal vertical plate with variable mass diffusion, in the presence of chemical reaction of first order. The dimensionless governing equations are solved using the Laplace-transform technique. The solutions are in terms of exponential and complementary error function. Such a study is found useful in magnetic control of molten iron flow in the steel industry, liquid metal cooling in nuclear reactors, magnetic suppression of molten semi-conducting materials and meteorology.

\section{GOVERNING EQUATIONS}

Consider the unsteady hydromagnetic flow of an electrically conducting fluid induced by viscous incompressible fluid past a uniformly accelerated motion of an isothermal vertical infinite plate when the fluid and the plate rotate as a rigid body with a uniform angular velocity $\Omega^{\prime}$ about $\mathrm{z}^{\prime}$-axis in the presence of an imposed uniform magnetic field $B_{0}$ normal to the plate. Initially, the temperature of the plate and concentration near the plate are assumed to be $\mathrm{T}_{\infty}$ and $\mathrm{C}_{\infty}^{\prime}$. At time $\mathrm{t}^{\prime}>0$, the plate starts moving with a velocity $\mathrm{u}=\mathrm{u}_{0} \mathrm{t}^{\prime}$ in its own plane and the temperature from the plate is raised to $\mathrm{Tw}$ and the concentration level near the plate are also raised linearly with time. Since the plate occupying the plane $z^{\prime}=0$ is of infinite extent, all the physical quantities depend only on $z^{\prime}$ and $t^{\prime}$. It is assumed that the induced magnetic field is negligible so that $=(0$, $\left.0, B_{0}\right)$. Then the unsteady flow is governed by freeconvective flow of an electrically conducting fluid in a rotating system under the usual Boussinesq's approximation in dimensionless form are as follows:

$$
\begin{aligned}
& \frac{\partial U}{\partial t}-2 \Omega V=G r \theta+G c C+\frac{\partial^{2} U}{\partial Z^{2}}-M U \\
& \frac{\partial V}{\partial t}+2 \Omega U=\frac{\partial^{2} V}{\partial Z^{2}}-M V \\
& \frac{\partial \theta}{\partial t}=\frac{1}{\operatorname{Pr}} \frac{\partial^{2} \theta}{\partial Z^{2}} \\
& \frac{\partial C}{\partial t}=\frac{1}{S c} \frac{\partial^{2} C}{\partial Z^{2}}-K C
\end{aligned}
$$

With the following initial and boundary conditions:

$\mathrm{u}=0, \quad \mathrm{~T}=\mathrm{T}_{\infty}, \mathrm{C}^{\prime}=\mathrm{C}_{\infty}^{\prime}$ for all $\mathrm{y}, \mathrm{t}^{\prime} \leq 0$ 
$\mathrm{u}=\mathrm{u}_{0} \mathrm{t}^{\prime}, \mathrm{T}=\mathrm{T}_{\mathrm{w}}, \mathrm{C}^{\prime}=\mathrm{C}^{\prime}+\left(\mathrm{C}_{\mathrm{w}}^{\prime}-\mathrm{C}^{\prime}\right) \mathrm{At}^{\prime}$

at $\mathrm{y}=0, \mathrm{t}^{\prime}>0$

$\mathrm{u} \rightarrow 0, \mathrm{~T} \rightarrow \mathrm{T}_{\infty}, \mathrm{C}^{\prime} \rightarrow \mathrm{C}_{\infty}^{\prime}$ as $\mathrm{y} \rightarrow \infty$

On introducing the following non-dimensional quantities:

$$
\begin{aligned}
& U=\frac{u}{\left(v u_{0}\right)^{1 / 3}}, V=\frac{v}{\left(v u_{0}\right)^{1 / 3}}, \\
& t=t^{\prime}\left(\frac{u_{0}^{2}}{v}\right)^{1 / 3}, Z=z\left(\frac{u_{0}}{v^{2}}\right)^{1 / 3}, \\
& \theta=\frac{T-T_{\infty}}{T_{w}-T_{\infty}}, G r=\frac{g \beta\left(T_{w}-T_{\infty}\right)}{u_{0}}, \\
& C=\frac{C^{\prime}-C_{\infty}^{\prime}}{C^{\prime}{ }_{w}^{\prime}-C_{\infty}^{\prime}}, G c=\frac{g \beta^{*}\left(C_{w}^{\prime}-C_{\infty}^{\prime}\right)}{u_{0}} \\
& M=\frac{\sigma B_{0}^{2}}{\rho}\left(\frac{v}{u_{0}^{2}}\right)^{1 / 3}, \operatorname{Pr}=\frac{\mu C_{p}}{k} \\
& S c=\frac{v}{D}, K=K_{l}\left(\frac{v}{u_{0}^{2}}\right)^{1 / 3} \\
& \text { where, } A=\left(\frac{u_{0}^{2}}{v}\right)^{1 / 3} \cdot
\end{aligned}
$$

The hydromagnetic rotating free-convection flow past an accelerated vertical plate is described by coupled partial differential Eq. (1) to Eq. (4) with the prescribed boundary conditions (5). To solve the Eq. (1) and Eq. (2), we introduce a complex velocity $\mathrm{q}=\mathrm{U}+\mathrm{i} \mathrm{V}$, Eq. (1) and Eq. (2) can be combined into a single equation:

$\frac{\partial q}{\partial t}=G r \theta+G c C+\frac{\partial^{2} q}{\partial Z^{2}}-m q$

The initial and boundary conditions in nondimensional quantities are

$\mathrm{q}=0, \quad \theta=0, \quad \mathrm{C}=0$, for all $\mathrm{Z}, \mathrm{t} \leq 0$

$\mathrm{t}>0: \quad \mathrm{q}=\mathrm{t}, \quad \theta=1, \mathrm{C}=\mathrm{t}$ at $\mathrm{Z}=0$

$\mathrm{q} \rightarrow 0, \theta \rightarrow 0, \mathrm{C} \rightarrow 0 \quad$ as $\quad \mathrm{Z} \rightarrow \infty$

where, $\mathrm{m}=\mathrm{M}+2 \mathrm{i} \Omega$.

\section{METHOD OF SOLUTION}

The dimensionless governing Eq. (3),(4) and (7), subject to the initial and boundary conditions (8), are solved by the usual Laplace-transform technique and the solutions are derived follows:

$$
\begin{aligned}
& q=\left(\frac{t}{2}+c+d+b d t\right) \\
& {\left[\begin{array}{l}
\exp (2 \eta \sqrt{m t}) \operatorname{erfc}(\eta+\sqrt{m t})+ \\
\exp (-2 \eta \sqrt{m t}) \operatorname{erfc}(\eta-\sqrt{m t})
\end{array}\right]} \\
& -\eta \sqrt{\frac{t}{m}}\left[\frac{1}{2}+b d\right] \\
& {\left[\begin{array}{l}
\exp (-2 \eta \sqrt{m t}) \operatorname{erfc}(\eta-\sqrt{m t}) \\
-\exp (2 \eta \sqrt{m t}) \operatorname{erfc}(\eta+\sqrt{m t})
\end{array}\right]} \\
& -2 c \operatorname{erfc}(\eta \sqrt{\operatorname{Pr}})-c \exp (a t) \\
& {\left[\begin{array}{l}
\exp (2 \eta \sqrt{(m+a) t}) \operatorname{erfc}(\eta+\sqrt{(m+a) t}) \\
+\exp (-2 \eta \sqrt{(m+a) t}) \operatorname{erfc}(\eta-\sqrt{(m+a) t})
\end{array}\right]} \\
& +c \exp (a t) \\
& {\left[\begin{array}{l}
\exp (2 \eta \sqrt{a t \operatorname{Pr}}) \operatorname{erfc}(\eta \sqrt{\operatorname{Pr}}+\sqrt{a t}) \\
+\exp (-2 \eta \sqrt{a t \operatorname{Pr}}) \operatorname{erfc}(\eta \sqrt{\operatorname{Pr}}-\sqrt{a t})
\end{array}\right]} \\
& -d \exp (b t) \\
& {\left[\begin{array}{c}
\exp (2 \eta \sqrt{(m+b) t}) \operatorname{erfc}(\eta+\sqrt{(m+b) t}) \\
+\exp (-2 \eta \sqrt{(m+b) t}) \operatorname{erfc}(\eta-\sqrt{(m+b) t})
\end{array}\right]} \\
& +d \exp (b t) \\
& {\left[\begin{array}{l}
\exp (-2 \eta \sqrt{S c(K+b) t}) \operatorname{erfc}(\eta \sqrt{S c}-\sqrt{(K+b) t}) \\
+\exp (2 \eta \sqrt{S c(K+b) t}) \operatorname{erfc}(\eta \sqrt{S c}+\sqrt{(K+b) t})
\end{array}\right]} \\
& +\frac{d b \eta \sqrt{S c t}}{\sqrt{K}} \\
& {\left[\begin{array}{l}
\exp (-2 \eta \sqrt{S c K t}) \operatorname{erfc}(\eta \sqrt{S c}-\sqrt{K t}) \\
-\exp (2 \eta \sqrt{S c K t}) \operatorname{erfc}(\eta \sqrt{S c}+\sqrt{K t})
\end{array}\right]} \\
& -d(1+b t) \\
& {\left[\begin{array}{l}
\exp (2 \eta \sqrt{S c K t}) \operatorname{erfc}(\eta \sqrt{S c}+\sqrt{K t}) \\
+\exp (-2 \eta \sqrt{S c K t}) \operatorname{erfc}(\eta \sqrt{S c}-\sqrt{K t})
\end{array}\right]} \\
& \theta=\operatorname{erfc}(\eta \sqrt{\operatorname{Pr}}) \\
& C=\frac{t}{2}\left[\begin{array}{l}
\exp (2 \eta \sqrt{S c K t}) \operatorname{erfc}(\eta \sqrt{S c}+\sqrt{K t}) \\
+\exp (-2 \eta \sqrt{S c K t}) \operatorname{erfc}(\eta \sqrt{S c}-\sqrt{K t})
\end{array}\right] \\
& -\frac{\eta \sqrt{S c t}}{2 \sqrt{K}} \\
& {\left[\begin{array}{l}
\exp (-2 \eta \sqrt{S c K t}) \operatorname{erfc}(\eta \sqrt{S c}-\sqrt{K t}) \\
-\exp (-2 \eta \sqrt{S c K t}) \operatorname{erfc}(\eta \sqrt{S c}+\sqrt{K t})
\end{array}\right]}
\end{aligned}
$$

where $\quad a=\frac{m}{\operatorname{Pr}-1}, \quad b=\frac{m-K S c}{S c-1}, \quad \eta=\frac{Z}{2 \sqrt{t}}$, $c=\frac{G r}{2 a(1-\mathrm{Pr})}, d=\frac{G c}{2 b^{2}(1-S c)}$.

In order to get the physical insight into the problem, the numerical values of $q$ have been computed from Eq. (9). While evaluating this expression, it is observed that the argument of the error function is complex and, hence, we have separated it into real and imaginary parts by using the following formula: 


$$
\begin{aligned}
& \text { erf }(a+i b)=\operatorname{erf}(a)+\frac{\exp \left(-a^{2}\right)}{2 a \pi} \\
& {[1-\cos (2 a b)+i \sin (2 a b)]} \\
& +\frac{2 \exp \left(-a^{2}\right)}{\pi} \\
& \sum_{n=1}^{\infty} \frac{\exp \left(-n^{2} / 4\right)}{n^{2}+4 a^{2}}\left[f_{n}(a, b)+i g_{n}(a, b)\right] \\
& +\in(a, b) \\
& \text { where } \\
& f_{n}=2 a-2 a \cosh (n b) \cos (2 a b) \\
& +n \sinh (n b) \sin (2 a b) \\
& g_{n}=2 a \cosh (n b) \sin (2 a b) \\
& +n \sinh (n b) \cos (2 a b) \\
& |\in(a, b)| \approx 10^{-16}|\operatorname{erf}(a+i b)|
\end{aligned}
$$

\section{RESUlts AND DisCUSSION}

For physical understanding of the problem, numerical computations are carried out for different physical parameters $\mathrm{Gr}, \mathrm{Gc}, \mathrm{Sc}, \mathrm{Pr}, \mathrm{m}$ and $\mathrm{t}$ upon the nature of the flow and transport. The value of the Schmidt number Sc is taken to be 0.6 which correspond to water-vapor. Also, the values of Prandtl number $\operatorname{Pr}$ are chosen such that they represent air $(\operatorname{Pr}=0.71)$ and water $(\operatorname{Pr}=7.0)$. The numerical values of the velocity, temperature and concentration fields are computed for different physical parameters like Prandtl number, rotation parameter, magnetic field parameter, thermal Grashof number, mass Grashof number, Schmidt number and time.

Figure 1 illustrates the effects of the magnetic field parameter on the velocity when $(\mathrm{M}=0,3,5), \mathrm{Gr}=$ $\mathrm{Gc}=5, \Omega=0.2, \mathrm{~K}=8, \operatorname{Pr}=7$ and $\mathrm{t}=0.6$. It is observed that the velocity increases with decreasing values of the magnetic field parameter. This shows that the increase in the magnetic field parameter leads to a fall in the velocity. This agrees with the expectations, since the magnetic field exerts a retarding force on the free convective flow.

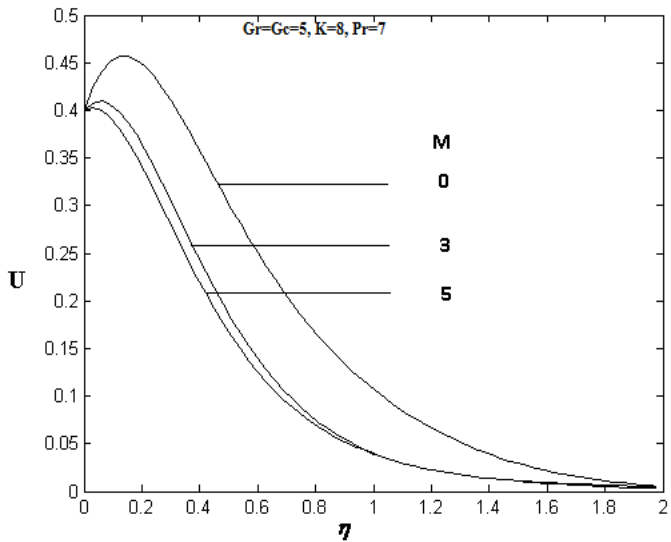

Fig. 1. Primary Velocity Profiles for different M

Figure 2 demonstrates the effects of different thermal Grashof number $(\mathrm{Gr}=2,5)$, mass Grashof number $(\mathrm{Gc}=2,5), \mathrm{M}=3, \mathrm{~K}=8, \Omega=0.2, \mathrm{Pr}=7$ on the primary velocity at time $t=0.2$. It is observed that the velocity increases with increasing values of the thermal Grashof number or mass Grashof number.

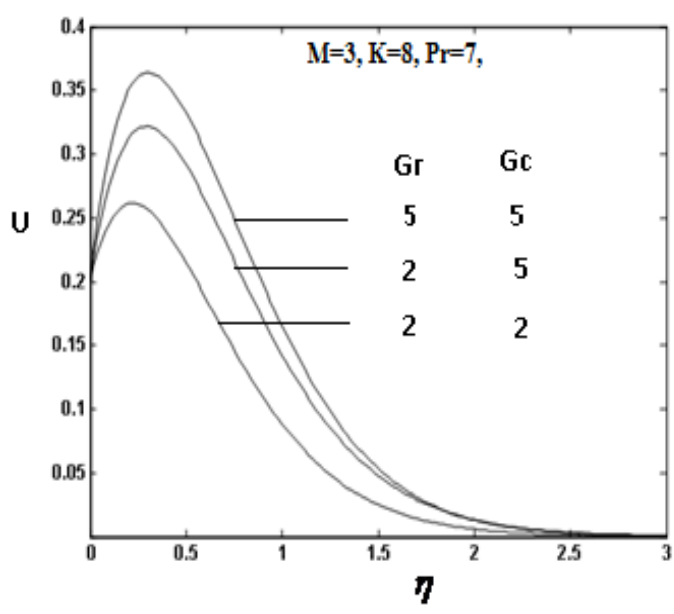

Fig. 2. Primary Velocity Profiles for different $\mathrm{Gr}$ and Gc

Figure 3 represents the effect of concentration profiles for different Schmidt number $(\mathrm{Sc}=0.16,0.3$, $0.6,2.01), \quad \mathrm{K}=0.2$ and $\mathrm{t}=0.2$. The effect of concentration is important in concentration field. The profiles have the common feature that the concentration decreases in a monotone fashion from the surface to a zero value far away in the free stream. It is observed that the wall concentration increases with decreasing values of the Schmidt number.

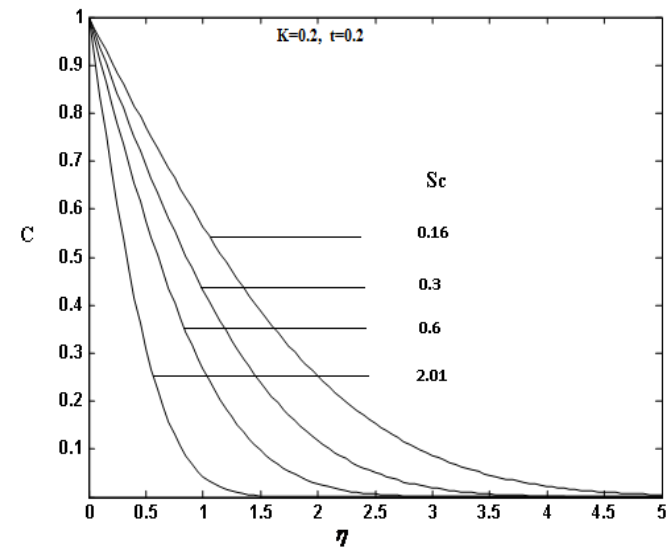

Fig. 3. Concentration Profiles for different $\mathrm{Sc}$

The temperature profiles are calculated for water and air from Eq. (10) and these are shown in Fig. 4. The effect of the Prandtl number plays an important role in temperature field. It is observed that the temperature increases with decreasing Prandtl number. This shows that the heat transfer is more in air than in water.

The concentration field for different values of chemical reaction parameter $(\mathrm{K}=0.2,2,5)$ and $\mathrm{t}=0.6$ are shown in Fig. 5. The trend shows that the wall concentration increases with decreasing values of the chemical reaction parameter $\mathrm{K}$. 


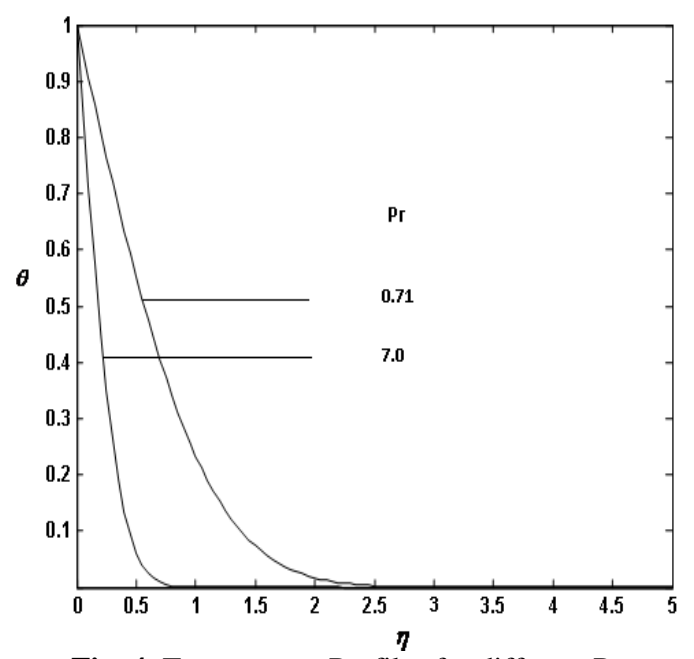

Fig. 4. Temperature Profiles for different $\operatorname{Pr}$

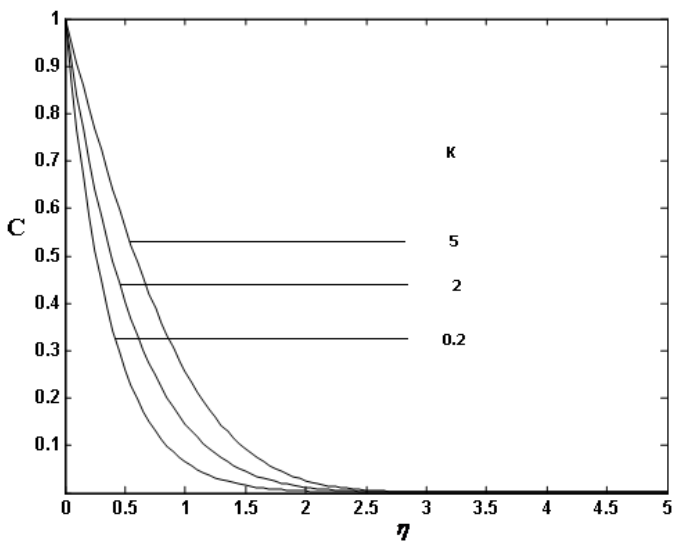

Fig. 5. Concentration Profiles for different $K$

The secondary velocity profiles for different thermal Grashof number $(\mathrm{Gr}=2,5)$, mass Grashof number $(\mathrm{Gc}=2,5), \mathrm{K}=8, \Omega=0.5, \mathrm{M}=10, \mathrm{Pr}=7$ and $\mathrm{t}=0.4$ are presented in Fig. 6 . The trend shows that the velocity decreases with increasing values of thermal Grahof number or mass Grashof number.

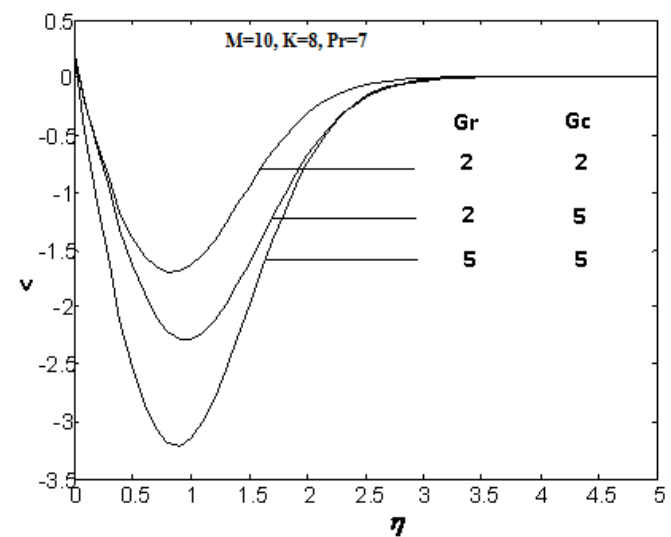

Fig. 6. Secondary Velocity Profiles for different Gr and Gc

The secondary velocity profiles for different rotation parameter $(\Omega=0.5,1), \mathrm{Gr}=\mathrm{Gc}=5, \mathrm{Pr}=7, \mathrm{~K}=8, \mathrm{M}$ $=3$ and $\mathrm{t}=0.2$ are shown in Fig. 7. It is observed that the velocity decreases with increasing values of the rotation parameter $\Omega$.

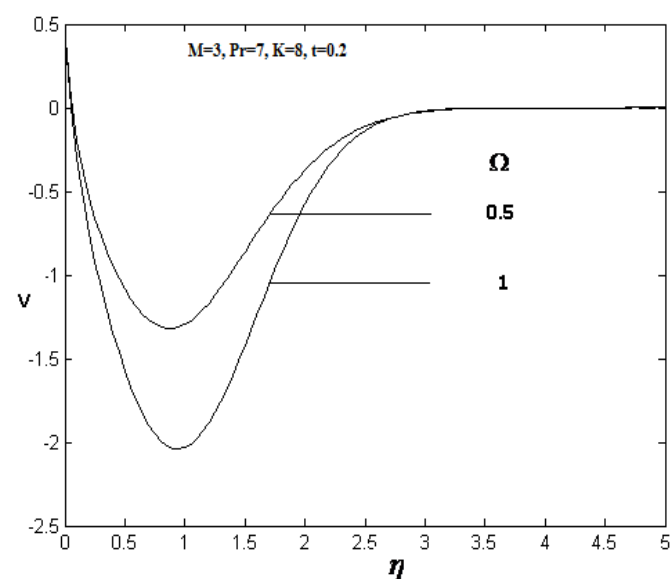

Fig. 7. Secondary Velocity Profiles for different $\Omega$

\section{CONCLUSION}

The theoretical solution of flow past a uniformly accelerated infinite isothermal vertical plate in the presence of variable mass diffusion has been studied. The dimensionless governing equations are solved by the usual Laplace transform technique. The effect of different parameters like thermal Grashof number, mass Grashof number and $t$ are studied graphically. It is observed that the velocity increases with increasing values of Gr, Gc and t. But the trend is just reversed with respect to the rotation parameter or magnetic field parameter $\mathrm{M}$.

\section{REFERENCES}

Basanth K.J. and R. Prasad (1990). Free convection and mass transfer effects on the flow past an accelerated vertical plate with heat sources, Mechanics Research Communications, 17, 143148.

Chambre P.L. and J.D. Young (1958). On the diffusion of a chemically reactive species in a laminar boundary layer flow. The Physics of Fluids, 1, 48-54.

Das U.N., R.K. Deka and V.M. Soundalgekar (1999). Effects of mass transfer on flow past an impulsively started infinite vertical plate with chemical reaction, The Bulletin of GUMA, 5, 1320.

Das U.N., R.K. Deka and V.M. Soundalgekar (1994). Effects of mass transfer on flow past an impulsively started infinite vertical plate with constant heat flux and chemical reaction. Forschung im Ingenieurwesen, 60, 284-287.

Gupta A.S., I. Pop and V.M. Soundalgekar (1979). Free convection effects on the flow past an accelerated vertical plate in an incompressible dissipative fluid, Rev. Roum. Sci. Techn.-Mec. Apl., 24 , 561-568. 
Kafousias N.G. and A. Raptis (1981). Mass transfer and free convection effects on the flow past an accelerated vertical infinite plate with variable suction or injection, Rev. Roum. Sci. Techn.Mec. Apl.. 26, 11-22.

Prasad V.R., R.N. Bhaskar, R. Muthucumaraswamy and B. Vasu (2011). Finite difference analysis of radiative free convection flow past an impulsively started vertical plate with variable heat and mass flux, Journal of Applied Fluid Mechanics, 4, 59-68.

Raptis A. and A.K. Singh (1981). MHD free convection flow past an accelerated vertical plate, Letters in Heat and Mass Transfer, 8, 137143.
Raptis A. and A.K. Singh (1985). Rotation effects on MHD free convection flow past an accelerated vertical plate, Mechanics Research Communications, 12 , 31-40.

Raptis A., G.J. Tzivanidis and C.P. Peridikis (1981). Hydromagnetic free convection flow past an accelerated vertical infinite plate with variable suction and heat flux, Letters in heat and mass transfer, 8, 137-143.

Singh A.K. (1984). Hydromagnetic free-convection flow past an impulsively started vertical plate in a rotating fluid, International Communications in Heat and Mass transfer, 11, 399-406.

Soundalgekar V.M. (1982). Effects of mass transfer on flow past a uniformly accelerated vertical plate, Letters in heat and mass transfer, 9, 6572. 